\title{
Shortest Path Embeddings of Graphs on Surfaces
}

\author{
Alfredo Hubard ${ }^{* 1}$, Vojtěch Kaluža ${ }^{\dagger 2}$, Arnaud de Mesmay ${ }^{\ddagger 3}$, and \\ Martin Tancer ${ }^{\S}$
}

1 INRIA Sophia-Antipolis and Université Paris-Est, Marne-la-Vallée, France

alfredo.hubard@inria.fr

2 Department of Applied Mathematics, Charles University, Prague, Czech

Republic

kaluza@kam.mff.cuni.cz

3 CNRS, Gipsa-Lab, Grenoble, France

arnaud.de-mesmay@gipsa-lab.fr

4 Department of Applied Mathematics and Institute of Theoretical Computer

Science, Charles University, Prague, Czech Republic

tancer@kam.mff.cuni.cz

\begin{abstract}
The classical theorem of Fáry states that every planar graph can be represented by an embedding in which every edge is represented by a straight line segment. We consider generalizations of Fáry's theorem to surfaces equipped with Riemannian metrics. In this setting, we require that every edge is drawn as a shortest path between its two endpoints and we call an embedding with this property a shortest path embedding. The main question addressed in this paper is whether given a closed surface $S$, there exists a Riemannian metric for which every topologically embeddable graph admits a shortest path embedding. This question is also motivated by various problems regarding crossing numbers on surfaces.

We observe that the round metrics on the sphere and the projective plane have this property. We provide flat metrics on the torus and the Klein bottle which also have this property.

Then we show that for the unit square flat metric on the Klein bottle there exists a graph without shortest path embeddings. We show, moreover, that for large $g$, there exist graphs $G$ embeddable into the orientable surface of genus $g$, such that with large probability a random hyperbolic metric does not admit a shortest path embedding of $G$, where the probability measure is proportional to the Weil-Petersson volume on moduli space.

Finally, we construct a hyperbolic metric on every orientable surface $S$ of genus $g$, such that every graph embeddable into $S$ can be embedded so that every edge is a concatenation of at most $O(g)$ shortest paths.
\end{abstract}

1998 ACM Subject Classification F.2.2 Nonnumerical Algorithms and Problems, G.2.2 Graph Theory, I.3.5 Computational Geometry and Object Modeling

Keywords and phrases Graph embedding, surface, shortest path, crossing number, hyperbolic geometry

Digital Object Identifier 10.4230/LIPIcs.SoCG.2016.43

\footnotetext{
* The research of A.H. was funded by GUDHI, geometric understanding in higher dimensions.

$\dagger$ V.K. was partially supported by the project CE-ITI (GAČR P202/12/G061).

$\ddagger$ The research of A.dM. leading to these results has received funding from the People Programme (Marie Curie Actions) of the European Union's Seventh Framework Programme (FP7/2007-2013) under REA grant agreement no. 291734.

$\S$ M. T. was partially supported by the project CE-ITI (GAČR P202/12/G061).
}

(c) (i) () Alfredo Hubard, Vojtěch Kaluža, Arnaud de Mesmay, and Martin Tancer;

32nd International Symposium on Computational Geometry (SoCG 2016).

Editors: Sándor Fekete and Anna Lubiw; Article No. 43; pp. 43:1-43:16

Leibniz International Proceedings in Informatics

LI I ICS Schloss Dagstuhl - Leibniz-Zentrum für Informatik, Dagstuhl Publishing, Germany 


\section{Introduction}

Fáry's theorem and joint crossing numbers. A famous theorem of Fáry [10] (originally showed by K. Wagner [33]) states that any simple planar graph can be embedded with straight line segments representing the edges. In this article, we investigate a generalization of Fáry's theorem to the setting of surface-embedded graphs. We focus on the following question: Given a surface $S$, is there a metric on $S$ such that every graph embeddable into $S$ admits a shortest path embedding, i.e., can be embedded so that the edges are represented by shortest paths? We call such a metric a universal shortest path metric. (We do not require that these shortest paths are unique but as we will see later on, in the case of our positive results, i.e., Theorem 1 and 4, the uniqueness of the shortest paths can be obtained as well.)

Before being enticed by this question, there were a number of problems involving joint embeddings of curves or graphs on surfaces, but arising from seemingly disparate settings, that motivated us to consider it. The literature on the subject goes back at least 15 years with Negami's work related to diagonal flips in triangulations [25]. He conjectured that there exists a universal constant $c$ such that for any pair of graphs $G_{1}$ and $G_{2}$ embedded in a surface $S$, there exists a homeomorphism $h: S \rightarrow S$ such that $h\left(G_{1}\right)$ and $G_{2}$ intersect transversely at their edges and the number of edge crossings satisfies $\operatorname{cr}\left(h\left(G_{1}\right), G_{2}\right) \leq c\left|E\left(G_{1}\right)\right| \cdot\left|E\left(G_{2}\right)\right|$. Recently, on one hand, Matoušek, Sedgwick, Tancer, and U. Wagner [20, 21], working on decidability of embeddability of 2-complexes into $\mathbb{R}^{3}$ and on the other hand, Geelen, Huynh, and Richter [12], in a quest for explicit bounds for graph minors, were faced with a similar question and provided bounds for related problems. Dually, this problem is equivalent to the one of finding a graph with a specific pattern within an embedded graph while bounding the multiplicity of the edges used: this is a fundamental question in computational topology of surfaces, where one is interested in finding objects with a fixed topology and minimal combinatorial complexity, e.g., short canonical systems of loops [19], short pants decompositions [5] or short octagonal decompositions [3]; see also [4].

Negami provided an upper bound on the number of crossings which grows linearly with $g$, and despite subsequent discoveries [1, 27], his conjecture is still open. In a paper that refines Negami's work [27], Richter and Salazar wrote "this [conjecture] seems eminently reasonable: why should two edges be forced to cross more than once?". The connection with our work is that if two graphs are embedded transversally by shortest path embeddings, then indeed no two edges cross more than once, since otherwise one of them could be shortcut.

Beyond these crossing numbers, the existence of shortest path universal metrics might be of interest to Riemannian geometers working on curvature free estimates and extremal metrics.

Related work. Generalizing Fáry's theorem is one of the drives of the field of graph drawing [31], where a lot of effort has been devoted towards embedding planar graphs with additional constraints such that the edges are straight lines, or polylines with few bends. Yet only few extensions to graphs embedded in surfaces are known. Two classical avatars of Fáry's theorem in the plane are of relevance to our work: Tutte's barycentric embedding theorem [32] and the Koebe-Andreev-Thurston circle packing theorem (see, for example, the book of Stephenson [28]). Both have been generalized to surfaces, providing positive answers to the following questions:

1. Given a surface $S$, a metric $m$, and a graph $G$ embeddable into $S$, can we embed the graph $G$ so that every edge is represented by a geodesic with respect to $m$ ?

2. Given a graph $G$ embeddable into $S$, does there exist a metric $m$ on $S$ so that $G$ embeds into $S$ with shortest paths? 


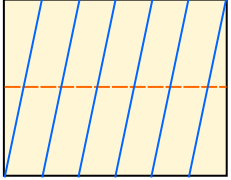

a.

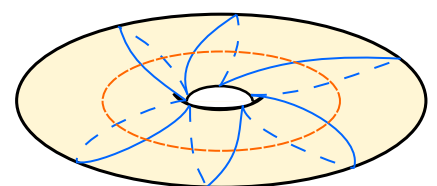

b.

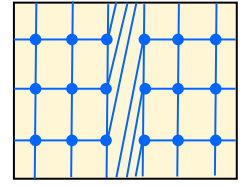

C.

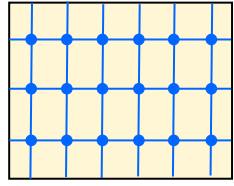

d.

Figure 1 a. and b. Two geodesics crossing many times. c. A grid embedded in a torus with geodesics. d. A reembedding of this grid with shortest paths.

Regarding the first question, Y. Colin de Verdière [6] generalized Tutte's barycentric embedding approach using a variational principle. The idea is to start with a topological embedding of the graph, replace the edges by springs, and let the system reach an equilibrium. Y. Colin de Verdière proved that for any metric of non-positive curvature, the edges become geodesics with disjoint interiors when the system reaches stability; moreover, this embedding is essentially unique within its homotopy class. However, geodesics need not be shortest paths, and two geodesics can intersect an arbitrarily large number of times, see Figure 1. Yet, these examples do not provide a negative answer to the second question, or to our main question, since we could change the embedding by a homeomorphism of the torus (thus even preserving the combinatorial map) to obtain a shortest path embedding.

The second question also has a positive answer, which can be proved via a generalization of the circle packing theorem to closed surfaces [28]. Namely, for every triangulation $T$ of a surface, there exists a metric of constant curvature so that $T$ can be represented as the contact graph of a family of circles. The representation of the triangulation that places a vertex at the center of its corresponding circle is an embedding with shortest paths. Such a representation can be computed efficiently and can be used as a tool for representing graphs on surfaces [23]. However, the metric is "chosen" by the triangulation, which makes this approach ill-suited for our purpose.

Our results. Our objective here is a mix of these last two results. On the one hand, we require shortest paths and not geodesics, on the other hand, we want a single metric for each surface and not one which depends on the triangulation. We will also consider the relaxation of our problem where we are allowed to use concatenations of shortest paths: we say that a metric is a $k$-universal shortest path metric if every topologically embeddable graph can be represented by an embedding in which edges are drawn as concatenations of $k$ shortest paths. This is akin to various problems in graph drawing where graphs are embedded with polylines with a bounded number of bends instead of straight lines [8, 30].

Our results and techniques are organized by curvature. We first observe that for the sphere and the projective plane, since there is a unique Riemannian metric of curvature 1, the circle packing approach applies to all graphs. Then, with the aid of irreducible triangulations, we provide flat metrics (i.e., of zero curvature) on the torus and the Klein bottle for which every graph admits a shortest path embedding.

- Theorem 1. The sphere $S^{2}$, the projective plane $\mathbb{R} P^{2}$, the torus $T^{2}$, and the Klein bottle $K$ can be endowed with a universal shortest path metric.

This result could lead to the idea that shortest path embeddings can be achieved for any metric, i.e., that every metric is a universal shortest path metric. We prove that this is not the case already for the unit square flat metric on the Klein bottle (arguably the first example to consider). 
- Theorem 2. Let $K$ denote the Klein bottle endowed with the unit square flat metric on the polygonal scheme $a b a^{-1} b$. Then there exists a graph embeddable into $K$ which cannot be embedded into $K$ so that the edges are shortest paths.

In higher genus, the number of irreducible triangulations is too large to check all cases by hand. Hyperbolic surfaces of large genus are hard to comprehend, but the probabilistic method allows us to show that if there exists universal shortest path metrics at all, their fraction tends to 0 as the genus tends to infinity.

- Theorem 3. For any $\varepsilon>0$, with probability tending to 1 as g goes to infinity, a random hyperbolic metric is not a $O\left(g^{1 / 3-\varepsilon}\right)$-universal shortest path metric. In particular, with probability tending to 1 as g goes to infinity, a random hyperbolic metric is not a universal shortest path metric.

We refer to Section 5 for an introduction to the probability measure used on the space of hyperbolic metrics, called the Weil-Petersson volume form. Our proof is an application of deep results on this volume form by Mirzakhani [22] and Guth, Parlier, and Young [14].

For genus $g>1$ we do not know if there exist shortest path universal metrics. But relaxing the question to concatenations of shortest paths and combining ideas from hyperbolic geometry and computational topology, we provide for every orientable surface of genus $g$ an $O(g)$-universal shortest paths metric. The proof relies on the octagonal decompositions of É. Colin de Verdière and Erickson [3] and a variant of the aforementioned theorem of Y. Colin de Verdière [6].

- Theorem 4. For every $g>1$, there exists an $O(g)$-universal shortest path hyperbolic metric $m$ on the orientable surface $S$ of genus $g$.

In this article we choose to focus on Riemannian metrics of constant curvature, but we remark that both of our last results also hold in the setting of piecewise-Euclidean metrics as well. For the upper bound, it suffices to replace hyperbolic hexagons with Euclidean ones, and the rest of the proof works similarly. For the lower bound it follows from the strong parallels between the Weil-Petersson volume form on moduli space and the counting measure on the space of $N=4 g$ Euclidean triangles randomly glued together. In particular the results that we use have analogs in this latter space: see Brooks and Makover [2] and the second half of the article of Guth, Parlier, and Young [14].

We have stated our results for graphs in this introduction. We note that one could consider the problem of shortest path embeddings for a graph with a fixed embedding up to a homeomorphism of the surface (i.e., for a combinatorial map), which is more in the spirit of Negami's conjecture. Our positive results can be stated in this stronger version; i.e., in our proofs the map is preserved. Our negative results would be weaker if the map had to be preserved, and in fact the proofs deal firstly with the statements for maps and then we derive the analog for graphs with some extra work.

The main open question is the existence of universal shortest path metrics, or $O(1)$ universal shortest path metrics. Natural candidates for these are given by certain celebrated extremal metrics, for instance the one minimizing $\int \log \operatorname{det}(\Delta) d s$, (where $\Delta$ denotes the Laplacian) that appeared in the work of Osgood, Phillips, and Sarnak [26], or the extremal ones in Gromov's systolic inequality [13].

After introducing the main definitions in Section 2, we will prove Theorems 1, 2, 3, and 4 in Sections 3, 4, 5, and 6, respectively. 


\section{Preliminaries}

In this article we only deal with compact surfaces without boundaries. By the classification theorem, these are characterized by their orientability and their genus, generally denoted by $g$. Orientable surfaces of genus 0 and 1 are respectively the sphere $S^{2}$ and the torus $T^{2}$, while non-orientable surfaces of genus 1 and 2 are the projective plane $\mathbb{R} P^{2}$ and the Klein bottle $K$. The orientable surface of genus $g$ is denoted by $S_{g}$. The Euler genus is equal to the genus for non-orientable surfaces and equals twice the genus for orientable surfaces.

By a path on a surface $S$ we mean a continuous map $p:[0,1] \rightarrow S$, and a closed curve denotes a continuous map $\gamma: S^{1} \rightarrow S$. These are simple if they are injective. We will be using occasionally the notions of homotopy, homology, and universal cover, we refer to Hatcher [15] for an introduction to these concepts. All the graphs that we consider in this paper are simple graphs unless specified otherwise, i.e., loops and multiple edges are disallowed. An embedding of a graph $G$ into a surface $S$ is, informally, a crossing-free drawing of $G$ on $S$. We refer to Mohar and Thomassen [24] for a thorough reference on graphs on surfaces, and only recall the main definitions. A graph embedding is cellular if its faces are homeomorphic to open disks. Euler's formula states that $v-e+f=2-g$ for any graph with $v$ vertices, $e$ edges, and $f$ faces cellularly embedded in a surface $S$ of Euler genus $g$. When the graph is not cellularly embedded, this becomes an inequality: $v-e+f \geq 2-g$. A triangulation of a surface is a cellular graph embedding such that all the faces are adjacent to three edges. By a slight abuse of language, we will sometimes refer to an embedding of a triangulation, by which we mean an embedding of its underlying graph which is homeomorphic to the given triangulation. A pants decomposition of a surface $S$ is a family of disjoint curves $\Gamma$ such that cutting $S$ along all of the curves of $\Gamma$ gives a disjoint union of pairs of pants, i.e., spheres with three boundaries. Every surface except the sphere, the projective plane, the torus, and the Klein bottle admits a pants decomposition with $3 g-3$ closed curves and $2 g-2$ pairs of pants. Note that all the pants decompositions are not topologically the same, i.e., are not related by a self-homeomorphism of the surface. A class of pants decompositions equivalent under such homeomorphisms will be called the (topological) type of the pants decomposition. We say that an embedding $f: G \rightarrow S$ contains a pants decomposition if there exists a subgraph $H \subseteq G$ such that $f: H \rightarrow S$ is a pants decomposition of $S$.

In this article, we will also be dealing with notions coming from Riemannian geometry, we refer to the book of do Carmo for more background [7]. By a metric we always mean a Riemannian metric, which associates to every point of a surface the curvature at this point. The Gauss-Bonnet theorem ties geometry and topology; it implies that the sign of a metric of constant curvature that a topological surface accepts is determined solely by its Euler genus.

A Riemannian metric induces a length functional on paths and closed curves. A path or a closed curve is a geodesic if the functional is locally minimal. Shortest paths between two points are global minima of the length functional. Unlike in the plane, geodesics are not, in general, shortest paths; in addition, neither geodesics nor shortest paths are unique in general. If we have a shortest path embedding of a graph where every edge is drawn as the unique shortest path between its endpoints, we speak of shortest paths embedding with uniqueness.

\section{Shortest path embeddings through minimal triangulations.}

- Theorem 1. The sphere $S^{2}$, the projective plane $\mathbb{R} P^{2}$, the torus $T^{2}$, and the Klein bottle $K$ can be endowed with a universal shortest path metric. 
In the theorem above, for $S^{2}$ and $\mathbb{R} P^{2}$ we use the round metric of positive constant curvature scaled to 1 . In the case of torus we use the flat metric obtained by the identification of the opposite edges of the square. In the case of the Klein bottle we can show that an analogous result fails with the flat square metric on the polygonal scheme $a b a^{-1} b$, as we will see in Section 4. But we can get the result for the metric obtained by the identification of the edges of a rectangle of dimensions $1 \times b$ where $b=\sqrt{4 / 3}+\varepsilon$ for some small $\varepsilon>0$. (The edges of length 1 are identified coherently, whereas the edges of length $b$ are identified in opposite directions.)

In all cases we can get shortest path embeddings with uniqueness. Actually, for the torus and the Klein bottle, uniqueness will be a convenient assumption for inductive proofs.

The sphere and the projective plane. The circle packing theorem states that any triangulation of the sphere can be represented as the contact graph of circles on the sphere [28, Theorem 4.3], endowed with the usual metric. Since any graph can be extended into a triangulation (adding new vertices if needed), and paths between centers of circles are shortest paths, this proves Theorem 1 for $S^{2}$. For $\mathbb{R} P^{2}$ endowed with the spherical metric, a similar circle packing theorem holds, yet we could not find a satisfying reference for it, so we provide a proof of it in the full version of the paper [16], based on building the circle-packing in the universal cover of $\mathbb{R} P^{2}$.

Minimal triangulations. Let $S$ be a surface and $T$ be a triangulation of it. The triangulation $T$ is called reducible, if it contains an edge $e$ such that the contraction of $e$ yields again a triangulation, which we denote by $T / e$. We refer to $e$ as a contractible edge (we do not mean contractibility in a topological sense). On the other hand, a triangulation is minimal (or irreducible), if no edge can be contracted this way. For every surface there is a finite list of minimal triangulations. In particular, for the torus $T^{2}$ this list consists of 21 triangulations found by Lawrencenko [17] and for the Klein bottle $K$ there are 29 minimal triangulations found by Sulanke [29].

The strategy of the proof of Theorem 1 for $T^{2}$ and $K$ is to show that it is sufficient to check Theorem 1 for minimal triangulations with appropriate fixed metric; see Lemma 5. Then, since every embedded graph can be extended to a triangulation (possibly with adding new vertices), we finish the proof by providing the list of shortest path embeddings of the minimal triangulations.

- Lemma 5. Let $S$ be a surface equipped with a flat metric. Let $T$ be a reducible triangulation with contractible edge $e$. Let us assume that $T / e$ admits a shortest path embedding with uniqueness into $S$. Then $T$ admits a shortest path embedding with uniqueness into $S$ as well.

The restriction on flat metrics in the lemma above does not seem essential, but this is all we need and this way the proof is quite simple.

Proof. Let $v$ be the vertex of $T / e$ obtained by the contraction of $e$. The idea is to consider the shortest path drawing of $T / e$. Then we perform the appropriate vertex splitting of $v$ (the inverse operation to the contraction) in a close neighborhood of $v$ so that we get a shortest path embedding of $T$.

In order to see that this is indeed possible, let us consider an edge $f=u v$ of $T / e$. Since the shortest paths are unique, a simple compactness argument shows that there is an $\varepsilon$-neighborhood $N_{f}$ of $f$ in $S$ which is isometric to an $\varepsilon$-neighborhood of a segment of the same length in $\mathbb{R}^{2}$ and such that for every $v^{\prime}$ in the $\varepsilon$-neighborhood of $v$, the straight line segment connecting $v$ and $v^{\prime}$ inside $N_{f}$ is the unique shortest path between $v$ and $v^{\prime}$ in $S$. 


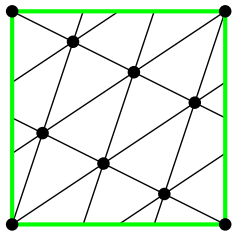

1

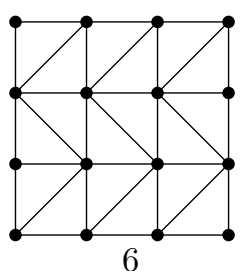

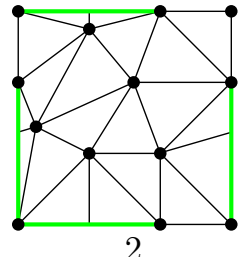

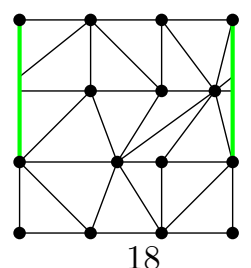

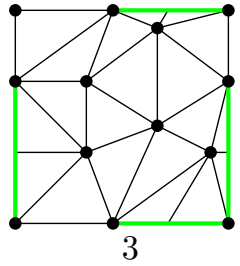

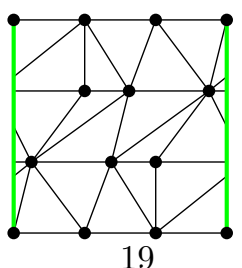

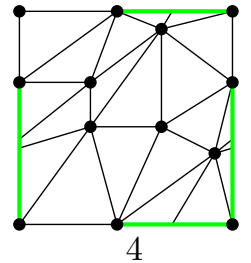

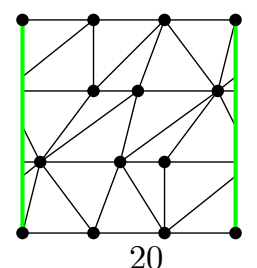

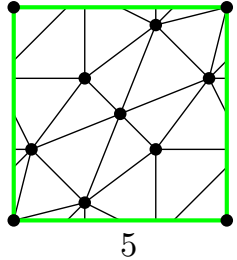

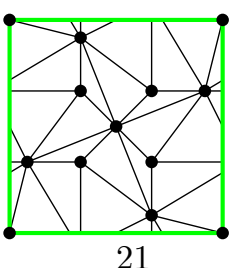

Figure 2 Minimal triangulations of the torus.

Therefore, considering such a neighborhood for every edge, it is sufficient to perform the vertex splitting of $v$ in sufficiently small neighborhood of $v$ so that we do not introduce new intersections as on the picture.

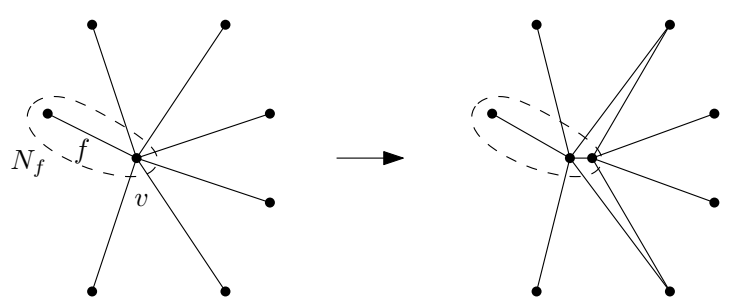

The minimal triangulations of $\boldsymbol{T}^{\mathbf{2}}$ and $\boldsymbol{K}$. In Figure 2 we provide a list of shortest path embeddings with uniqueness of minimal triangulations of the torus with a flat metric obtained by identifying the opposite edges of the unit square. They are in the same order as in the book of Mohar and Thomassen [24, Figure 5.3]. The black (thin) edges are the edges of the triangulation whereas the green (thick) edges are the identified boundaries of the unit square which are not parts of the edges of the triangulations. We just skip drawings of the triangulations 7 to 17 , because they are all analogous to the triangulation 6 , they only have different patterns of diagonals. It is clear that every edge is a geodesic. In order to check that each of them is drawn as a shortest path, it is sufficient to verify that each edge projects vertically and horizontally to a segment of length less than $\frac{1}{2}$.

For the Klein bottle $K$, we also provide a metric such that all the minimal triangulations admit shortest path embeddings with uniqueness. We obtain this metric as the identification of the edges of the rectangle $R=[0, a] \times[0, b]$, where $a=1$ and $b=\sqrt{4 / 3}+\varepsilon$ for sufficiently small $\varepsilon$. The edges of length 1 are identified in coherent directions. The edges of length $b$ are identified in the opposite directions. Minimal triangulations of the Klein bottle were obtained by Lawrencenko and Negami [18], and Sulanke [29], and we show how to embed them with shortest paths in Figure 3. The details regarding these embeddings are explained in the full version [16], but for a cursory glance the Figures 3 should be self-explanatory with a couple of remarks. In some cases an additional shift is necessary by a small value $\frac{1}{1000}$ (but this value is large compared to $\varepsilon$ ): this is indicated by arrows next to the vertices. (The pair of arrows in Kh25 indicates a shift by $\frac{2}{1000}$.) The triangulations split into two 


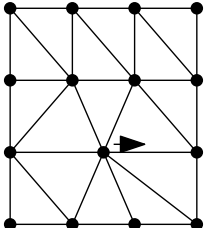

Kh1

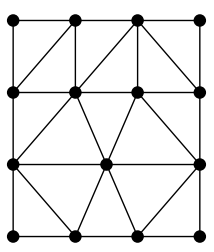

Kh6

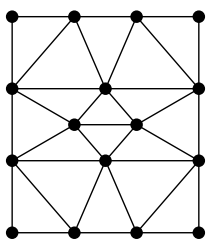

Kh11

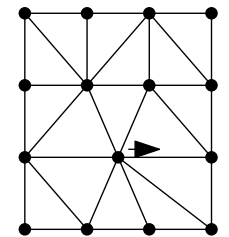

Kh2

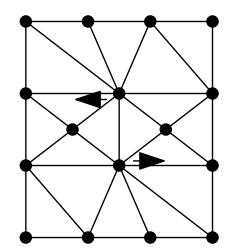

$\mathrm{Kh} 7$

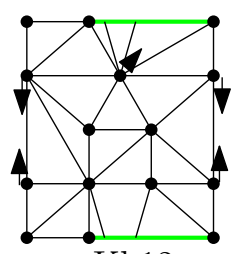

Kh12

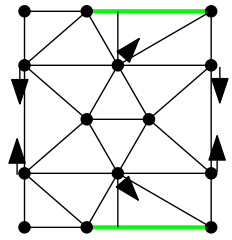

Kh3

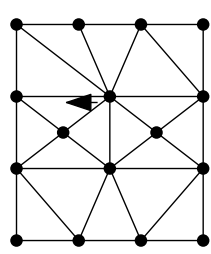

Kh8

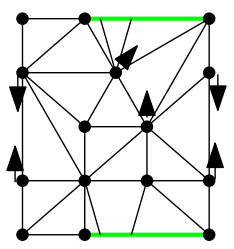

Kh13

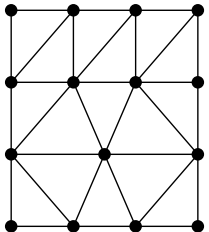

Kh4
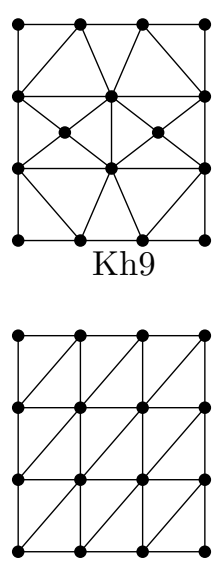

Kh14

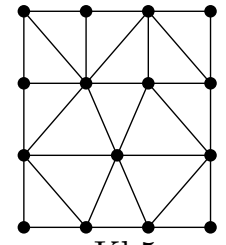

Kh5
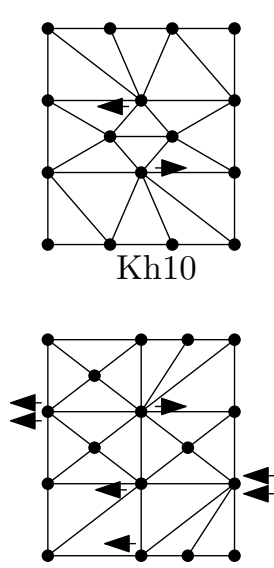

Kh25

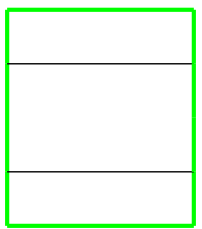

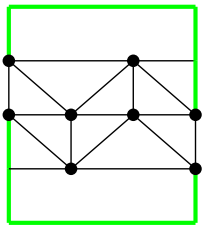

Mb1

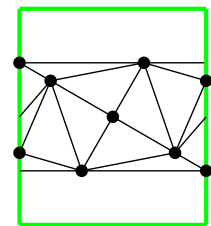

Mb2

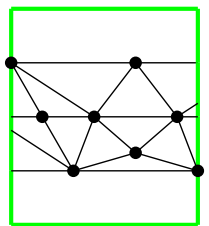

Mb3

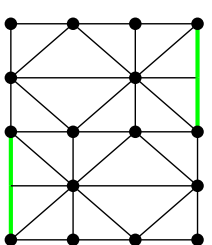

$\mathrm{Kc1}$

Figure 3 Minimal triangulations of the Klein bottle.

types - so called Kh cases and Kc cases. For the Kh cases we omit few pictures analogous to Kh14. For the Kc cases, the triangulations of the Klein bottle are obtained by gluing together two triangulations of the Möbius band pictured in the bottom line; Kc1, obtained from two copies of Mb1, is provided as an example.

\section{Square flat metric on the Klein bottle}

The task of this section is to prove the following theorem.

- Theorem 2. Let $K$ denote the Klein bottle endowed with the unit square flat metric on the polygonal scheme $a b a^{-1} b$. Then there exists a graph embeddable into $K$ which cannot be embedded into $K$ so that the edges are shortest paths.

We consider the minimal triangulation Kc1 (see Figure 3) and we denote by $G$ the underlying graph for this triangulation. We will prove that $G$ does not admit a shortest path embedding into $K$ with the square metric. First, we observe that the triangulation Kc1 is the only embedding of $G$ into $K$. The proof is given in the full version [16]. 

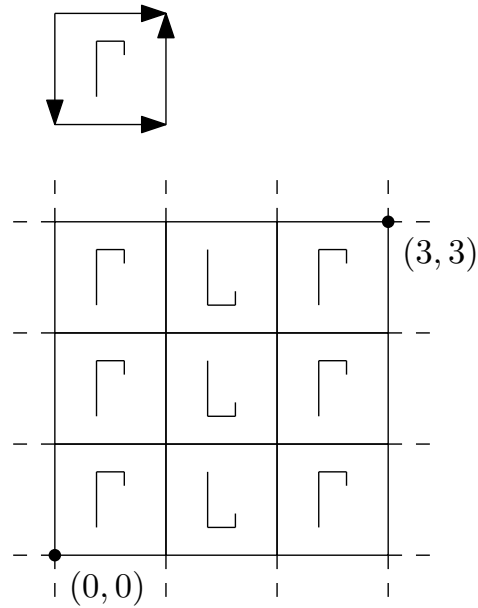
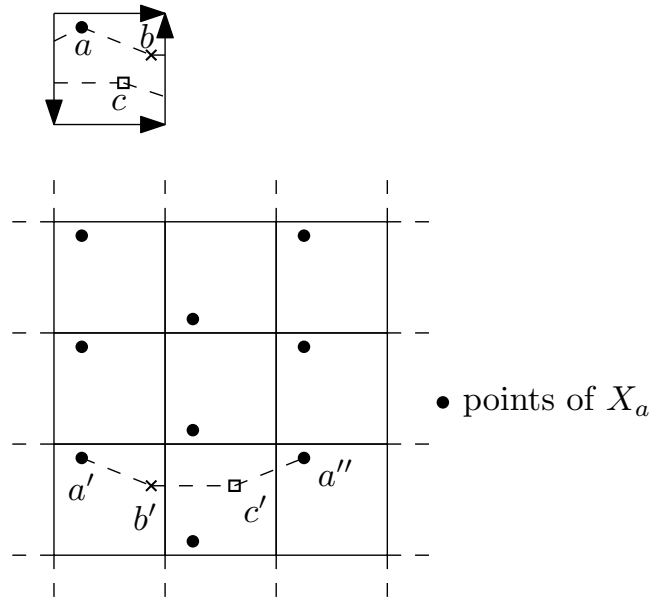

Figure 4 The Klein bottle with a letter ' $\Gamma$ ', and its universal cover (left). A lift of the cycle $a b c$ (right).

- Proposition 6. G has a unique embedding into the Klein bottle.

For contradiction, let us assume that $G$ admits a shortest paths embedding into $K$. We know that Kc1 is obtained by gluing two triangulations of a Möbius band along a cycle of length 3 (the triangle corresponding to this cycle is not part of the triangulation). Let $a b c$ be this cycle. With a slight abuse of notation we identify this cycle with its image in the (hypothetical) shortest path embedding into $K$. Our strategy is to show that already $a b c$ cannot be embedded into $K$ with shortest path edges, which will give the required contradiction. By Proposition 6, we know that $a b c$ splits $K$ to two Möbius bands.

Let $X=\mathbb{R}^{2}$ be the universal cover of $K$ (with standard Euclidean metric). Let $\pi: X \rightarrow K$ be the isometric projection corresponding to the cover. We will represent the Klein bottle with the flat-square metric as the unit square $[0,1]^{2}$ with suitable identification of the edges $\left(a b a^{-1} b\right.$, as in the previous section). We will use the convention that $\pi\left((0,1)^{2}\right)=(0,1)^{2}$; that is, the projection is the identity on the interior of this square. See Figure 4.

Given a point $p \in K$ we set $X_{p}:=\pi^{-1}(p)$. Finally, let $\mathcal{V}_{p}$ be the Voronoi diagram in $X$ corresponding to the set $X_{p}$.

Lemma 7. Let $p$ and $q$ be two points in $K$ and $\gamma$ be an arc (edge) connecting them, considered as a subset of $K$. Then $\gamma$ is the unique shortest path between $p$ and $q$ if and only if there are $p^{\prime} \in X_{p}, q^{\prime} \in X_{q}$ such that $\gamma=\pi\left(\overline{p^{\prime} q^{\prime}}\right)$ where $\overline{p^{\prime} q^{\prime}}$ denotes the straight edge connecting $p^{\prime}$ and $q^{\prime}$ in $X$ and $q^{\prime}$ belongs to the open Voronoi cell for $p^{\prime}$ in $\mathcal{V}_{p}$.

The proof directly follows the correspondence between $K$ and $X$. It is given in the full version [16].

Now let us lift the cycle $a b c$ to a path $a^{\prime} b^{\prime} c^{\prime} a^{\prime \prime}$ in $X$; see Figure 4 . Given a curve in $X$, we call the length of its projection to the $x$-axis, the "horizontal length" of the curve; similarly we speak about the horizontal distance and the vertical distance of two points in $X$.

Lemma 8. The horizontal distance between $a^{\prime}$ and $a^{\prime \prime}$ is at least 2.

Sketch of a proof. If we consider the point $a^{\prime}$ fixed, then the position of $a^{\prime \prime}$ in $X_{a}$ determines the homotopy class of the cycle $a b c$ in the fundamental group $\pi_{1}(K)$. 
The horizontal distance between $a^{\prime}$ and $a^{\prime \prime}$ must be a non-negative integer. However, by checking the homology class of $a b c$ in the $\mathbb{Z}$ and $\mathbb{Z}_{2}$-homology, it is possible to rule out the cases when this distance is 0 . By the fact that $a b c$ is a double-sided cycle, it is possible to rule out the distance 1 . The full proof is given in the full version of this paper [16].

- Lemma 9. Let $\gamma$ be a unique shortest path in $K$ connecting points $p$ and $q$. Let $\gamma^{\prime}$ be a lift of $\gamma$ with endpoints $p^{\prime}$ and $q^{\prime}$. Then the horizontal distance in $X$ between $p^{\prime}$ and $q^{\prime}$ is less than $\frac{5}{8}$.

Proof. Let $C$ be the open Voronoi cell for $p^{\prime}$ in $\mathcal{V}_{p}$. By Lemma $7, q^{\prime}$ belongs to $C$. Therefore, it is sufficient to check that every point $c^{\prime}$ of $C$ has horizontal distance less than $\frac{5}{8}$ from $p^{\prime}$. Without loss of generality, we may assume that the $x$-coordinate of $p^{\prime}$ equals 0 since shifting $p^{\prime}$ in horizontal direction only shifts $X_{p}$ and $\mathcal{V}_{p}$ ( note that this is not true for the vertical direction). For contradiction, there is a $c^{\prime}$ in $C$ at distance at least $\frac{5}{8}$ and without loss of generality the $x$-coordinate of $c^{\prime}$ is positive. Let $p^{\prime \prime}$ be the point of $X_{p}$ with $x$-coordinate equal 1 which is vertically closest to $c^{\prime}$ (pick any suitable point in case of draw); see the picture on the left. The vertical distance between $c^{\prime}$ and $p^{\prime \prime}$ is at most $\frac{1}{2}$. A simple calculation, using the Pythagoras theorem, gives that $p^{\prime \prime}$ is at most as far from $c^{\prime}$ as $p^{\prime}$. A contradiction.

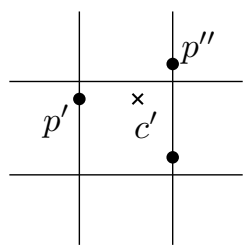

Finally, we summarize how the previous lemmas yield a contradiction. By Lemma 8, the horizontal distance between $a^{\prime}$ and $a^{\prime \prime}$ is at least 2. On the other hand, Lemma 9 gives that the horizontal length of each of the edges $a^{\prime} b^{\prime}, b^{\prime} c^{\prime}$, and $c^{\prime} a^{\prime \prime}$ is at most $\frac{5}{8}$, altogether at most $\frac{15}{8}$. This gives the required contradiction, which finishes the proof of Theorem 2 .

\section{Asymptotically almost all hyperbolic metrics are not universal}

Before stating the main theorem of this section, we will give some very quick background on the geometry of surfaces, we refer to Farb and Margalit [9] for a proper introduction. The Teichmüller space $\mathcal{T}_{g}$ of a surface $S$ of genus $g$ denotes the set of hyperbolic metrics on $S$, such that two metrics are equivalent if they are related by an isometry isotopic to the identity. In some contexts, like ours, one might also want to identify metrics related by an isometry (not necessarily isotopic to the identity). The corresponding space is called the moduli space $\mathcal{M}_{g}$ of the surface, and is obtained by quotienting $\mathcal{T}_{g}$ by the mapping class group of $S$, i.e., its group of homeomorphisms. This moduli space can be endowed with multiple structures, here we will be interested in a particular one, called the Weil-Petersson metric. This metric provides $\mathcal{M}_{g}$ with a Riemannian structure of finite volume, and therefore by renormalizing, we obtain a probability space, allowing to choose a random metric. We can now state the main theorem of this section.

- Theorem 3. For any $\varepsilon>0$, with probability tending to 1 as g goes to infinity, a random hyperbolic metric is not a $O\left(g^{1 / 3-\varepsilon}\right)$-universal shortest path metric. In particular, with probability tending to 1 as g goes to infinity, a random hyperbolic metric is not a universal shortest path metric. 
The proof is a consequence of two important results on random hyperbolic metrics. The first is a small variant of a theorem of Guth, Parlier, and Young [14, Theorem 1]. Before stating it, we need some definitions.

Given a hyperbolic metric $m$ on a surface $S$, we say that $m$ has total pants length at least $\ell$ if in any pants decomposition $\Gamma$ of $S$, the lengths of the closed curves of $\Gamma$ sum up to at least $\ell$. We say that $m$ has total pants length of type $\xi$ at least $\ell$ if in any pants decomposition $\Gamma$ of $S$ of type $\xi$, the lengths of the closed curves of $\Gamma$ sum up to at least $\ell$.

- Theorem 10. For any $\varepsilon>0$, a random metric on $\mathcal{M}_{g}$ has total pants length at least $g^{7 / 6-\varepsilon}$ with probability tending to 1 as $g \rightarrow \infty$. For any $\varepsilon>0$ and any family of types of pants decomposition $\xi_{g}$, a random metric on $\mathcal{M}_{g}$ has total pants length of fixed type $\xi_{g}$ at least $g^{4 / 3-\varepsilon}$ with probability tending to 1 as $g \rightarrow \infty$.

The proof is almost identical to the one in Guth, Parlier, and Young [14]; it is summarized in the full version of this paper [16].

The following is an immediate corollary of this theorem.

- Corollary 11. Let $T_{g}$ be a family of triangulations of $S_{g}$, such that every member of $T_{g}$ contains a pants decomposition of fixed type $\xi_{g}$. For any $\varepsilon>0$, with probability tending to 1 as $g \rightarrow \infty$, a shortest embedding of $T_{g}$ into a random hyperbolic surface of genus $g$ has length at least $\Omega\left(g^{4 / 3-\varepsilon}\right)$.

The next theorem was proved by Mirzakhani [22, Theorem 4.10].

- Theorem 12. With probability tending to 1 , the diameter of a random hyperbolic surface of genus $g$ is $O(\log g)$.

Theorem 3 is proved by providing an explicit family of graphs $G_{g}$ which will embed badly. It is defined in the following way for $g \geq 2$. Let $\xi_{g}$ be a type of pants decompositions for every value of $g$.

- We start with a pants decomposition of type $\xi_{g}$ of a surface $S_{g}$

- We place four vertices on every boundary curve.

- We triangulate each pair of pants with a bounded size triangulation so that each cycle of length 3 bounds a triangle in the triangulation, and any path connecting two boundary components of the pair of pants has length at least 4 (in particular $G_{g}$ is a simple graph and each cycle of length 3 in the graph $G_{g}$ bounds a triangle in the triangulation).

The following proposition controls the issues related to the flexibility of embeddings of graphs into surfaces, it is proved in the full version [16].

- Proposition 13. There is a unique embedding of $G_{g}$ into $S_{g}$, up to a homeomorphism; in particular every embedding contains a pants decomposition of type $\xi_{g}$.

With these three results at hand we are ready to provide a proof of the theorem.

Proof of Theorem 3. We use the family of graphs $G_{g}$ previously defined. Since there are $O(g)$ curves in a pants decomposition, it contains $O(g)$ edges, and every embedding of $G_{g}$ into $S_{g}$ contains a pants decomposition of type $\xi_{g}$ by Proposition 13 .

Now, by Corollary 11, for every $\varepsilon>0$, and for $g$ large enough, the probability that the shortest possible embedding of $G_{g}$ into a random metric has length at least $O\left(g^{4 / 3-\varepsilon}\right)$ is at least $1-\varepsilon / 2$. In particular, since there are $O(g)$ edges in $G_{g}$, some edge $e_{g}$ in this embedding must have length at least $\Omega\left(g^{1 / 3-\varepsilon}\right)$. By Theorem 12, we can choose $g$ large enough so that with probability at least $1-\frac{\varepsilon}{2}$, the random hyperbolic metric has diameter $O(\log g)$. Hence, 

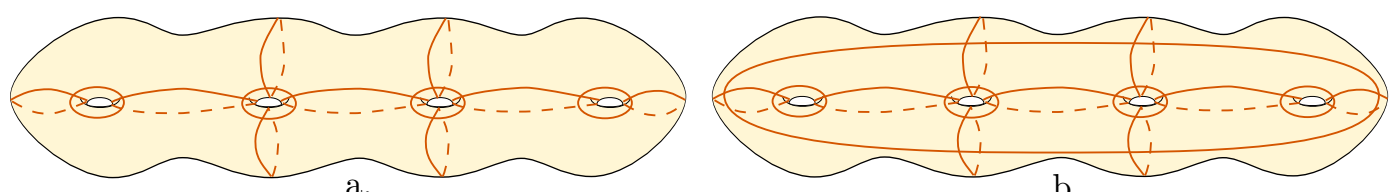

b.

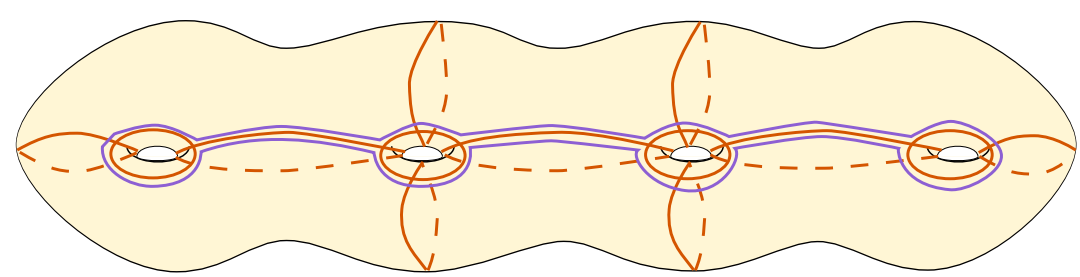

c.

Figure 5 a. An octagonal decomposition b. A hexagonal decomposition c. How to add one closed curve to upgrade an octagonal decomposition to a hexagonal decomposition.

by the union bound, with probability $1-\varepsilon$ both properties hold. Therefore, for every $\varepsilon>0$, there exists some value $g_{0}$ such that for any $g \geq g_{0}$, in any embedding of $G_{g}$, there exists an edge $e_{g}=(x, y)$ such that $\ell_{m}\left(e_{g}\right)=\Omega\left(g^{1 / 3-\varepsilon}\right)$, but $d_{m}(x, y) \leq \operatorname{diam}(m) \leq O(\log g)$. This implies that $e$ is not drawn by a shortest path. Similarly, subdividing each edge $O\left(g^{1 / 3-\varepsilon}\right)$ times will run into the same issue. This concludes the proof.

\section{Higher genus: positive results}

- Theorem 4. For every $g>1$, there exists an $O(g)$-universal shortest path hyperbolic metric $m$ on the orientable surface $S$ of genus $g$.

Our approach to prove Theorem 4 is to cut the surface $S_{g}$ with a hexagonal decomposition $\Delta$, so that every edge of $G$ is cut $O(g)$ times by this decomposition $\Delta$. The construction to do this is a slight modification of the octagonal decompositions provided by É. Colin de Verdière and Erickson [3, Theorem 3.1]. Each of the hexagons is then endowed with a specific hyperbolic metric $m_{H}$, and pasting these together yields the hyperbolic metric $m$ on $S_{g}$. The hyperbolic metric $m_{H}$ is chosen so that the hexagons are convex, i.e., the shortest paths between points of a hexagon stay within this hexagon. Therefore, there only remains to embed the graph $G$ cut along $\Delta$, separately in every hexagon with shortest paths. To do this, we use a variant of a theorem of Y. Colin de Verdière [6] which generalizes Tutte's barycentric method to metrics of nonpositive curvature.

Hexagonal decompositions. A hexagonal decomposition, respectively an octagonal decomposition of $S_{g}$ is an arrangement of closed curves on $S_{g}$ that is homeomorphic to the one pictured in Figure 5.a., respectively Figure 5.b. In particular, every vertex has degree four and every face has six sides, respectively eight sides.

Octagonal decompositions were introduced by É. Colin de Verdière and Erickson [3] where they showed how to compute one that does not cross the edges of an embedded graph too many times. We restate their theorem in our language.

Theorem 14 ([3, Theorem 3.1]). Let $G$ be a graph embedded in a surface $S_{g}$ for $g \geq 2$. There exists an octagonal decomposition $\Gamma$ of $S_{g}$ such that each edge of $G$ crosses each closed curve of $\Gamma$ a constant number of times. 


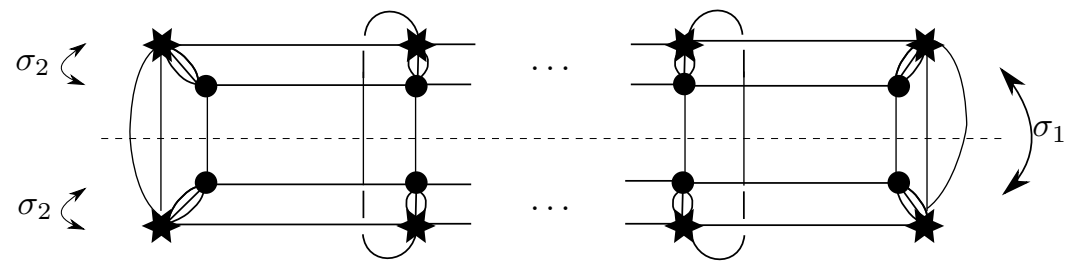

Figure 6 The intersection graph $I$ and the two involutions: $\sigma_{1}$ is the symmetry about the dashed horizontal line, and $\sigma_{2}$ swaps every disk and its adjacent star.

We observe that this octagonal decomposition can be upgraded to a hexagonal decomposition that still does not cross $G$ too much:

Corollary 15. Let $G$ be a graph embedded in a surface $S_{g}$. There exists a hexagonal decomposition $\Delta$ of $S_{g}$ such that each edge of $G$ crosses each closed curve of $\Delta$ a constant number of times, except for maybe one closed curve which is allowed to cross each edge of $G$ at most $O(g)$ times. In particular, the number of crossing between every edge of $G$ and $\Delta$ is $O(g)$.

Proof. The decomposition $\Delta$ is simply obtained by taking the decomposition $\Gamma$ and adding a single curve that follows closely a concatenation of $O(g)$ subpaths of curves of $\Gamma$, see Figure 5c. The resulting arrangement of curves has the topology of a hexagonal decomposition, and the bounds on the number of crossings results directly from the construction.

The hyperbolic metric. We first endow each hexagon of the hexagonal decomposition with the hyperbolic metric $m_{H}$ of an equilateral right-angled hyperbolic hexagon. Since the hexagons have right angles and the vertices of a hexagonal decomposition have degree 4 , this metric can be safely pasted between hexagons to endow $S_{g}$ with a hyperbolic metric $m$. The main property of this metric that we will use is the following one:

- Proposition 16. Every hexagon $H$, viewed as a subset of $S_{g}$ endowed with $m$, is convex, i.e., every path between $x, y \in H$ that is a shortest path in $H$ is also a shortest path in $S_{g}$.

Proof. The proof relies on an exchange argument based on the symmetries of the hexagonal decomposition.

The intersection graph $I$ of the hexagonal decomposition is defined by taking one vertex for each hexagon and edges between adjacent hexagons (we allow multiple edges). We are interested in two graph automorphisms which are also involutions. These are pictured in Figure 6:

- The symmetry $\sigma_{1}$ about the horizontal axis, corresponding to the so-called hyperelliptic involution of the surface $S$.

- The automorphism $\sigma_{2}$ swapping every hexagon with its neighbor in the octagonal decomposition.

Since all the hexagons are isometric, these involutions correspond naturally to isometric involutions of $S$.

Now, let $\gamma$ be a shortest path between two vertices $x$ and $y$ in a hexagon $H_{1}$, let us assume without loss of generality that $H_{1}$ is in the upper part of $I$. This path $\gamma$ naturally induces a walk in $I$ obtained by taking each hexagon of which interior is met by $\gamma$. This walk does not backtrack at some hexagon $H$ : otherwise one could shortcut $\gamma$ by staying on the boundary of $H$. 
From $\gamma$, one can build a path $\gamma^{\prime}$ between $x$ and $y$ which stays in the upper half of the graph: for every maximal subpath of the graph in the lower half, one applies the isometry $\sigma_{1}$, effectively mirroring these paths in the upper half. Similarly, by applying $\sigma_{2}$, one obtains a path $\gamma^{\prime \prime}$, which only uses half of the hexagons. The walk in $I$ corresponding to $\gamma^{\prime \prime}$ lies now in a path. Since it does not backtrack, it is necessarily trivial and never leaves hexagon $H_{1}$. We have thus found a shortest path in $H_{1}$ connecting $x$ and $y$.

Finishing the proof. We prove in this paragraph how to reembed a graph embedded in a hexagon so that its edges are shortest paths. This allows us to finish the proof.

- Theorem 17. Let $G$ be a graph embedded as a triangulation in a hyperbolic hexagon $H$ endowed with the metric $m_{H}$. If there are no dividing edges in $G$, i.e., edges between two non-adjacent vertices on the boundary of $H$, then $G$ can be embedded with geodesics, with the vertices on the boundary of $H$ in the same positions as in the initial embedding.

Given this theorem, we can now conclude the proof of Theorem 4: the intersections of our input graph with the hexagonal decompositions subdivide it, and in each hexagon one can subdivide the dividing edges if needed, then upgrade the subgraph to a triangulation, and finally embed it with this theorem. The details are in the full version [16]. We note that by subdividing each edge once more, the shortest paths we obtain are unique.

The proof of Theorem 17 is obtained in a spirit similar to the proof of the one of the celebrated spring theorem of Tutte [32]. However, there are two main differences which prevent us from directly appealing to the literature: on the one hand the metric is not Euclidean but hyperbolic, and on the other hand the boundary of the input polygon is not strictly convex, since there may be multiple vertices of $G$ on a geodesic boundary of $H$. The hypothesis on dividing edges is tailored to circumvent the second issue, and in a Euclidean setting it was proved by Floater [11] that the correspond embedding theorem holds. Regarding the first issue, Y. Colin de Verdière stated a Tutte embedding theorem $[6$, Theorem 3] for the hyperbolic setting with strictly convex boundary, yet he actually did not provide a proof for it. In Appendix A of the full version [16] we show how to prove Theorem 17 in the generality that we need following the ideas laid out (in French) by Y. Colin de Verdière in the rest of his article [6].

Acknowledgements. We are grateful to Éric Colin de Verdière for his involvement in the early stages of this research, to Sergio Cabello, Francis Lazarus, Bojan Mohar, and Eric Sedgwick for helpful discussions and to Xavier Goaoc for organizing the workshop that led to this work.

\section{References}

1 Dan Archdeacon and C. Paul Bonnington. Two maps on one surface. Journal of Graph Theory, 36(4):198-216, 2001.

2 Robert Brooks and Eran Makover. Random construction of Riemann surfaces. J. Differential Geom., 68(1):121-157, 2004.

3 Éric Colin de Verdière and Jeff Erickson. Tightening nonsimple paths and cycles on surfaces. SIAM Journal on Computing, 39(8):3784-3813, 2010.

4 Éric Colin de Verdière, Alfredo Hubard, and Arnaud de Mesmay. Discrete systolic inequalities and decompositions of triangulated surfaces. Discrete $\mathscr{B}$ Computational Geometry, 53(3):587-620, 2015. 
5 Éric Colin de Verdière and Francis Lazarus. Optimal pants decompositions and shortest homotopic cycles on an orientable surface. Journal of the ACM, 54(4):Article 18, 2007.

6 Yves Colin de Verdière. Comment rendre géodésique une triangulation d'une surface ? L'Enseignement Mathématique, 37:201-212, 1991.

7 Manfredo P. do Carmo. Riemannian geometry. Birkhäuser, 1992.

8 Cesim Erten and Stephen G. Kobourov. Simultaneous embedding of planar graphs with few bends. Journal of Graph Algorithms and Applications, 9(3):347-364, 2005.

9 Benson Farb and Dan Margalit. A primer on mapping class groups. Princeton University Press, 2011.

10 István Fáry. On straight line representations of planar graphs. Acta scientiarum mathematicarum (Szeged), 11:229-233, 1948.

11 Michael Floater. One-to-one piecewise linear mappings over triangulations. Mathematics of Computation, 72(242):685-696, 2003.

12 Jim Geelen, Tony Huynh, and R. Bruce Richter. Explicit bounds for graph minors. arXiv:1305.1451, 2013.

13 Mikhael Gromov. Filling Riemannian manifolds. Journal of Differential Geometry, 1983.

14 Larry Guth, Hugo Parlier, and Robert Young. Pants decompositions of random surfaces. Geometric and Functional Analysis, 21:1069-1090, 2011.

15 Allen Hatcher. Algebraic topology. Cambridge University Press, 2002. Available at http: //www. math. cornell.edu/ hatcher/.

16 Alfredo Hubard, Vojtěch Kaluža, Arnaud de Mesmay, and Martin Tancer. Shortest path embeddings of graphs on surfaces. arXiv preprint: http://arxiv.org/abs/1602.067r8, 2016.

17 Serge Lawrencenko. The irreducible triangulations of the torus. Ukrainski $\breve{i}$ Geometricheskii Sbornik, 30:52-62, 1987.

18 Serge Lawrencenko and Seiya Negami. Irreducible triangulations of the Klein bottle. Journal of Combinatorial Theory, Series B, 70(2):265-291, 1997.

19 Francis Lazarus, Michel Pocchiola, Gert Vegter, and Anne Verroust. Computing a canonical polygonal schema of an orientable triangulated surface. In Proceedings of the 17th Annual Symposium on Computational Geometry (SOCG), pages 80-89. ACM, 2001.

20 Jiř́ Matoušek, Eric Sedgwick, Martin Tancer, and Uli Wagner. Untangling two systems of noncrossing curves. In Stephen Wismath and Alexander Wolff, editors, Graph Drawing volume 8242 of Lecture Notes in Computer Science, pages 472-483. Springer International Publishing, 2013.

21 Jiří Matoušek, Eric Sedgwick, Martin Tancer, and Uli Wagner. Embeddability in the 3sphere is decidable. In Proceedings of the Thirtieth Annual Symposium on Computational Geometry, SOCG'14, pages 78-84. ACM, 2014.

22 Maryam Mirzakhani. Growth of Weil-Petersson volumes and random hyperbolic surface of large genus. J. Differential Geom., 94(2):267-300, 062013.

23 Bojan Mohar. Drawing graphs in the hyperbolic plane. In Graph Drawing, pages 127-136. Springer, 1999.

24 Bojan Mohar and Carsten Thomassen. Graphs on surfaces. Johns Hopkins Studies in the Mathematical Sciences. Johns Hopkins University Press, 2001.

25 Seiya Negami. Crossing numbers of graph embedding pairs on closed surfaces. Journal of Graph Theory, 36(1):8-23, 2001.

26 B. Osgood, R. Phillips, and P. Sarnak. Extremals of determinants of Laplacians. Journal of Functional Analysis, 80(1):148-211, 1988.

27 R. Bruce Richter and Gelasio Salazar. Two maps with large representativity on one surface. Journal of Graph Theory, 50(3):234-245, 2005.

28 Kenneth Stephenson. Introduction to circle packing: The theory of discrete analytic functions. Cambridge University Press, 2005. 
29 Thom Sulanke. Note on the irreducible triangulations of the Klein bottle. Journal of Combinatorial Theory, Series B, 96:964-972, 2006.

30 Roberto Tamassia. On embedding a graph in the grid with the minimum number of bends. SIAM Journal on Computing, 16(3):421-444, 1987.

31 Roberto Tamassia. Handbook of graph drawing and visualization. CRC press, 2013.

32 William T. Tutte. How to draw a graph. Proceedings of the London Mathematical Society, 13:743-768, 1963.

33 K. Wagner. Bemerkungen zum Vierfarbenproblem. Jahresbericht der Deutschen Mathematiker-Vereinigung, 46:26-32, 1936. 